\title{
Nerve-Sparing Radical Hysterectomy - An Old Japanese Concept
}

\author{
Nicolae Bacalbaşa ${ }^{1}$, Beatrice Lintoiu ${ }^{2}$
}

Corresponding author:

Nicolae Bacalbașa, M.D.

Dimitrie Racoviță Street, no. 2,

Bucharest, Romania

E-mail: nicolae_bacalbasa@yahoo.ro

\author{
1"Carol Davila" University of Medicine and Pharmacy, Bucharest, Romania \\ 2"Profesor Dr. Agripa Ionescu” Clinical Emergency Hospital, Bucharest, Romania
}

\section{ABSTRACT}

Conventional radical hysterectomy, the main part of the standard surgical treatment in cervical cancer, causes damage to the pelvic autonomic nerves, thus leading to urinary, anorectal and sexual dysfunctions. Many researches have focused on surgical approaches that protect the pelvic nerves in order to decrease postoperative related morbidity. Nervesparing radical hysterectomy is a safe technique that improves the quality of life of patients who undergo cervical cancer surgery without compromising oncologic outcomes.

Key words: radical hysterectomy, nerve-sparing radical hysterectomy, urinary dysfunction

\section{INTRODUCTION}

Cervical cancer is the second most common cause of cancer mortality in women worldwide (1).

Conventional surgical management of cervical cancer (CC) is radical hysterectomy $(\mathrm{RH})$ with pelvic lymphadenectomy (2).

Conventional surgery is associated with postoperative bladder dysfunctions, colorectal motility disorders and sexual dysfunctions, a consequence of accidental damage to the pelvic autonomic nerves during surgery. Improving surgical treatment as well as postoperative quality of life is increasingly important challenges given that more than $54 \%$ of women diagnosed with CC are younger than 50 years (3). Thus, nerve-sparing radical hysterectomy (NSRH) has emerged in the last 30 years for reducing surgery-related dysfunctions without compromising oncologic outcomes.

\section{Classification of radical hysterectomies}

Classification of radical hysterectomies is very important for interpretation of outcomes and for establishing a standard treatment. The pioneers of the principles of radicality for the cervical cancer, combining the concept of the organ extended surgery and the concept of the lymphatic territory surgery, are the Viennese surgeon Ernst Wertheim and the Romanian surgeon Thoma 
Ionescu, who have presented their view in 1902, at the International Congress of Surgery and Gynecology in Rome.

The concept of nerve sparing is an old Japanese concept. In 1921, H. Okabayashi published a surgical technique, developed with his professor, S. Takayama, which had as particularity, the preservation of the nerve plexus (4).

In 1961, Kobayashi, at Tokyo University, sustained the concept of the nerve-sparing radical hysterectomy by demonstrating that the cardinal ligament is composed of two parts: a vascular part, which is the ventral part, containing the deep uterine vein, and a neural part. He also demonstrated that pelvic splanchnic nerves runs along the most dorsal part of the cardinal ligament (3).

In 1974, Piver-Rutledge-Smith (5) divided radical hysterectomies into 5 classes, a classification respected by many surgeons. Nevertheless, over time, this classification became outdated and has several disadvantages: doesn't take into account the "Terminologia Anatomica", ignores the nerve-sparing approach, mixes the lateral, dorsal and vaginal extension of the resections, has no clear limits between the classes, class I is not radical.

In the recent years, the concept of NSRH, i.e. the systematic preservation of the pelvic nerve system, including the hypogastric nerves, the pelvic splanchnic nerves, the pelvic plexus and the vesical branches of the pelvic plexus, was better established. Querleu and Morrow (6) published in 2008 another classification of the radical hysterectomies in Lancet Oncology magazine, one that is gaining more and more followers. It defines four main categories according to extent of removal of paracervix and uses only reliable anatomic landmarks: ureter, internal iliac vessels and pelvic wall. It includes the nerve-sparing techniques and the new concept of paracervical lymphadenectomy. Type A - a total removal of the cervix and a minimal removal of the paracervix, which is transected lateral to the cervix, but medial to the ureter; bladder and rectal pillars are transected close to the uterus; the vagina is cut less than $1 \mathrm{~cm}$; type B- the ureter is unroofed and rolled laterally, thus allowing the transection of paracervix at level of ureteral tunnel; neural component of paracervix is not transected; bladder and rectal pillars are resected at a distance from the uterus. In B2 subtype there is an additional lateral paracervical lymph node dissection; at least $1 \mathrm{~cm}$ of he vagina is resected; type $\mathrm{C}$ - resection of the paracervix at pelvic sidewall, resection of the entire paracervix, at junction with hypogastric vessels; resection of bladder pillar at bladder and rectal pillar at rectum; ureter is completely mobilised. C1- nerve sparing resection of medial and lateral components of bladder pillar, the cranial and caudal components of paracervix; there is no transection of the uterosacral ligaments at the pelvic sidewall. C2-the neural part is not left untouched, so the paracervix is transected lower than the deep uterine vein, which is used as a landmark between $\mathrm{C} 1$ and $\mathrm{C} 2$. Type D-exenterative procedures: D1-resection of the entire paracervix at the level of the pelvic sidewall along with the hypogastric vessels, exposing the roots of the sciatic nerve. D2- removes also adjacent fascia and muscular structures of the pelvic sidewall. The splanchnic nerves are at risk withcaudal extension of surgery beyond C2 (dorsal parametriumat the level of the deep uterine vein) and dorsal extension ofsurgery beyond $\mathrm{D} 1$.

\section{Pelvic neuroanatomy and sites of injury}

The autonomic pelvic nerves includes hypogastric plexus and nerves, splanchnic nerves, and visceral branches. The hypogastric nerves join the pelvic nerve plexus from the superior ventral side. The pelvic splanchnic nerves, which originated from the ventral rami S2 to S4, join from the lateral dorsal side (7).

There is the sympathetic system with the superior hypogastric plexus and hypogastric nerves and the parasympathetic system with the pelvic splanchnic nerves. They anastomose at the level of the inferior hypogastric plexus and after that there are visceral nerve branches for the bladder, rectum and vagina. Regarding their function on the bladder and sexual functions, the sympathetic system favour compliance and storage by stimulating the smooth urethral sphincter and inhibiting the detrusor muscle and the parasympathetic system favour voiding by stimulating detrusor while relaxing the smooth urethral sphincter and also favour vaginal lubrification and genital swelling.

Underneath the promontory, the superior hypogastric plexus (SHP) divides into the hypogastric nerves, which run craniocaudally and $2 \mathrm{~cm}$ mediodorsally to the ureters, in the posterior and lateral layer of the uterosacral ligaments (USL). The pelvic splanchnic nerves are mainly parasympathetic and are involved with detrusor contractility, rectal function, and vaginal lubrication. They form the inferior hypogastric plexus (IHP) along with the hypogastric nerves and some sacral splanchnic nerves. The IHP is a laminar structure with a triangular shape with a sagital direction in the pararectal fossa and is constituted by both sympathetic and parasympathetic fibers. The plexus stretches from anarea anterolateral to the rectum to the lateral vaginal 
wall and bladder base, supplying the genital tract, bladder, urethra, and most of the rectum. IHP is formed from nerves that arise from three different sources: from the SHP via the hypogastric nerve, from the sympathetic trunk via sacral splanchnic nerves, and from the ventral rami of sacral spinal nerves via pelvic splanchnic nerves. Parasympathetic contributions for the IHP arrive from sacral rami of more inferior levels after crossing the pararectal space. Locations in the female hemipelvis in which we canidentify nerve fibers from IHP are as follows: into the USL, parametrially, pararectally, and in a few cases, in the fold between the urinary bladder and the uterus. From the anterior part of the IHP, several groups of fibers are coursed to the pelvic organs and according to their location, we can identify three groups of nerve fibers: a) superior branches, which mostly comming with the ureter and are responsible for the innervation of the urinary bladder; b) central branches, which follow the course of the uterine artery to the uterine cervix; and c) inferior branches, which innervate the inferior part of the rectum and form the neurovascular bundle to the erectile tissue. Vesical efferences have many variations, but most commonly there are two kinds of fibers: ones satellite of the ureter, outside and below of it and another group just under the paracervical tissue, passing through vesicouterine ligament, utero-vesical junction to the bladder wall lateral and medial $(8,9,10)$.

Injury of the pelvic autonomic nerves may occur at different steps during radical hysterectomy (11): the superior hypogastric plexus at presacral and periaortic lymph node dissection, the hypogastric nerves at resection of the rectouterineligaments, the proximal inferior hypogastric plexus at divisionof the uterosacral and cardinal ligaments, the distal inferior hypogastric plexus at divisionvesicouterine ligament $(11,12,13)$.

In a conventional nerve-sparing procedure, the parametrium and paracolpium tissue should be transected just above the hypogastric nerves, allowing the pelvic nerve plexus to be preserved. Consequently, exposure of the pelvic splanchnic nerves is not necessary. In contrast, during the radical nerve-sparing procedure, the hypogastric nerves are sacrificed and the pelvic nerve plexus is partially preserved to attain more radicality than with the conventional nervesparing procedure. Therefore, the denudation of pelvic splanchnic nerves is necessary $(14,15)$.

Lesions of the superior hypogastric plexus and hypogastric nerves induce urinary incontinence and urgency; lesions of the pelvic splanchnic nerves generate bladder atonia, disorders of bladder sensitivity, decrease blood flow to vagina and lubrication; injuries to the inferior hypogastric plexus and visceral branches generate various dysfunctions according to the level of injury. Consequences seem to be more limited if unilateral injury, even if there are important variation in hypogastric nerves in about $25 \%$ of women (7).

\section{Nerve-sparing radical hysterectomy - technical aspects}

Radical hysterectomy, that is, complete removal of the uterus and parametrium, is a basic surgical procedure for early uterine cervical cancer, but also for advanced stage. A concept of the parametrium that should be removed or how deeply and widely the parametrium should be dissected has varied significantly with the time. Bladder dysfunction after RH has been well documented, damage to the pelvic nerve plexus (inferior hypogastric plexus) and its vesical branches the pathways for neurogenic bladder control, being the main cause of dysfunction.

To preserve the bladder function after radical hysterectomy several authors have described procedures for NSRH. The hypogastric nerves constitute the main anatomical landmark of NSRH because they form the upper limit of the pelvic nerve plexus and the vesical branches $(14,15)$.

For the surgical procedure, surgeons usually provide two spaces by blunt dissection in the small pelvis, that is, the pararectal and paravesical spaces. Thereby, the cardinal ligament of the uterus, a specific part of the parametrium, is developed between these two spaces. Many surgeons believed that in combination with complete removal of the uterus, entire parts of the cardinal ligament and sacro-uterine ligament should be removed, including their deep extension to the pelvic floor. The Tokyo method, developed originally by Ogino (1950) and revised by Kobayashi (1961) and Sakamoto and Takizawa (1988) demonstrated that the cardinal ligament is divided into two parts, the superficial or ventral "vascular part" as a target for ligation and removal and the deep or dorsal "nerve part" that should be preserved because it is likely to contain the pelvic splanchnic nerves. Many Western and Asian gynecologists seem to accept the Tokyo method as a standard method for nerve-sparing radical hysterectomy $(2,3,7,16)$.

A simplified technique of nerve-sparing radical hysterectomy was described by Li. B et al (17) in 2011, the key point of the procedure being to integrally preserve the nerve plane (meso-ureter and its extension) without meticulous dissection of the detailed nerve structure: 


\section{Division of the uterosacral ligament}

Along with the ureter, the meso-ureter (the proximal part of the nerve plane), containing the hypogastric nerve, is lateralized from the posterior layer of the broad ligament. Okabayashi's space between the meso-ureter and the uterosacral ligament is developed by blunt dissection. The prerectal space is developed by transversely incising the peritoneum of the pouch of Douglas. The uterosacral ligament is divided near its root, and the proximal part of the nerve plane is preserved.

\section{Division of the cardinal ligament}

The paravesical space and pararectal space are developed by blunt dissection. After transecting the uterine artery and the superficial uterine vein from its origin, lymphatic fatty tissue around the deep uterine vein is removed, then the pelvic splanchnic nerve branches underneath the deep uterine vein are kept intact.

\section{Transection of the uterine branch}

of the inferior hypogastric plexus

The ureter is completely unroofed and freed from its tunnel. A loose avascular area (the fourth space) between the posterior leaf of the vesicouterine ligament and the paracolpium is developed by carefully blunt dissection. Along with the parametrial tissue, the uterine branch is transected between the fourth space and Okabayashi's space. The distal part of the nerve plane (extension of the meso-ureter), containing the bladder branches, is lateralized.

\section{Division of the posterior leaf}

of the vesicouterine ligament

Between the vesicouterine pouch and the fourth space, the posterior leaf of the vesicouterine ligament is transected before the uterus is removed. The nerve plane right underneath the ureter is integrally preserved without any unnecessary dissection $(18,19)$.

To preserve the bladder innervations, two main surgical rules must be respected: to identify the hypogastric nerve before entering the cardinal ligament and to maintain a perfect hemostasis throughout all the surgical steps. The nerve thus can be maintained under direct vision, making possible its preservation.

Recently, Höckel et al. (20) developed the concept of total mesometrial resection (TMMR) for the treatment of cervical cancer. This concept is based on the fact that local tumor spread might be restricted within the Müllerian morphogenetic unit from which the tumor originates. Therefore, en-bloc resection of the Müllerian morphogenetic unit, including the uterus, proximal vagina and mesometrium, might lead to more radical resection and better local control even without the need for adjuvant radiotherapy. The authors also proposed that the main pelvic autonomic nerves, which are in a different tissue unit, should be separated from the Müllerian morphogenetic unit and integrally preserved. The pelvic nerve plane seemed to be well preserved by this procedure. However, the anatomy and technique of TMMR are complicated.

As for the lenght of the vaginal resection, BenedettiPanici et al (21) have suggested that the incidence of lower urinary tract dysfunction is related to the extent of resection of the rectovaginal ligaments and vaginal tissue.In the NSRH, the level of colpectomy has been restricted to $2 \mathrm{~cm}$ to preserve the most distal part of the vesicovaginal branch of the inferior hypogastric plexus that courses medially along the lateral vaginal wall to the bladder. In contrast, satisfactory results were obtained by Sakuragi et al (12) even with a length of the resected vagina ranging from 2 to $4.5 \mathrm{~cm}$.

Obesity can induce technical difficulty in the performance of the nerve-sparing technique because the operating field is deep, visibility is poor and the presence of considerable amount of fatty tissue surrounding the nerve structures can obscure the delicate nerve tissues (16).

\section{Radicality of NSRH}

Concerning the oncological efficacy, nonrandomized studies on the survival of patients operated on with the nerve-sparing technique reported relapse and survival rates similar to the classical radical hysterectomy $(22,23)$. Sakuragi et al $(5)$ reported cumulative DFS rates of $95.5 \%$ for NSRH and $100 \%$ for RH at 24 months, while van den Tillaart et al (23) observed similar 5-year OS and local recurrence rates within 24 months for NSRH and $\mathrm{RH}$.

The finding that NSRH and $\mathrm{RH}$ are associated with similar survival may reflect the fact that they are associated with a similar extent of resection, parametrial width and vaginal cuff lengh. This is contrary to the belief among some clinicians that NSRH involves less extensive resection and therefore can lead to lower survival and higher risk of recurrence. Putambeker et al (2) reported 5-year DFS rates of $92 \%$ for IB1 CC and 78\% for IB2 CC, with corresponding 5-year OS rates of $96 \%$ and $83 \%$. The authors concluded that NSRH does not compromise surgical radicality. 


\section{Urinary tract dysfunction after radical hysterectomy}

Conventional radical surgery is known to cause urinary dysfunctions, such as stress incontinence, sensory loss and bladder voiding dysfunction as hypotonic and hypertonic detrusor function in $12 \%$ to $85 \%$. These symptoms may affect the quality of life of patients surviving cervical cancer (24).

Many reports assess bladder function after NSRH by measuring the residual volume of urine after voiding. Benedetti-Panici et al (21) described that only $15 \%$ of patients who underwent $\mathrm{RH}$ had a residual volume more than $30 \%$ (considered abnormal) of their bladder capacity. However, $76 \%$ of patients developed bladder dysfunction during the 12 months after surgery. Therefore, measuring the residual volume of urine after voiding might not be sufficient to evaluate bladder function. Urodynamic evaluation is necessary to ascertain bladder dysfunction after NSRH $(19,21)$.

Urinary bladder dysfunctions represent the most frequent type of morbidity after radical hysterectomy $(\mathrm{RH})$ and the impairment of spontaneous voiding represents the most prominent symptom in the early postoperative period. The mean time to spontaneous recovery differs significantly in published studies from 9 to 31 days. It is hypothesized that perivesical edema, autonomic denervation, and/or loss of urinary bladder support have a role in the etiology of theimpairment, but the pathophysiology remains incompletely understood.

The study of Cibula D. et al (25) retrospectively analyzed data from 85 patients (67 radical hysterectomy, 6 radical parametrectomy and 12 radical trachelectomy), of which 35 underwent nerve-sparing modification, 19 underwent type $C$ radicality of procedure and 31 type $\mathrm{D}$ radicality of procedure. Multivariate analysis revealed three significant parameters: procedure radicality, type of procedure (radical hysterectomy vs radical trachelectomy), and a negative correlation with body mass index. Radicality of parametrectomy was the most significant parameter influencing the interval to spontaneous voiding recovery; significant differences were observed between nerve-sparing and type $D$ procedures. An interesting finding was the better functional outcomes after radical trachelectomy, which is likely caused by the preservation of supportive structures after the fertility-sparing procedure. Other parameters, including tumor volume, operation time, number of harvested lymph nodes, age, surgeon, method of bladder drainage or adjuvant radiotherapy, were not significant.
A significantly shorter time to spontaneous voiding (defined as urine residuum less than $30 \mathrm{~mL}$ ) was described by Possover et al (26) as early as in 2000 in 38 patients after NSRH in comparison with 28 patients who had undergone RH type 3, 11 versus 21 days. In their study, the principle of nerve-sparing modification focused only on the preservation of splanchnic nerves in the caudal part of lateral parametria, whereas nowadays, it is the preservation of hypogastric plexus that is considered essential.

Kanao $\mathrm{H}$ et al (19) conducted a study on 53 patients to investigate the detailed anatomy of the pelvic nerve plexus and its vesical branches and to study the correlation between preserved pelvic nerve networks and bladder function using urodynamic studies performed before surgery and at 1,3,6 and 12 months after surgery. The evaluation of sensory and motor function of the bladder in this study required two parameters for uro-dynamic analysis: first desire to void (FDV) used to evaluate sensory function and PdetQmax, the detrusor contraction pressure at maximum flow, used to evaluate motor function. For the nerve sparing group, the recovery of sensory and motor functions was significantly higher within 12 months after surgery, and at 6 months and 12 months statistically higher than that at 1 month after surgery. So, the preserved nerve function can be improved within 12 months after surgery. The authors suggested that the distributions of sensory nerves and motor nerves differ, the sensory nerves being distributed predominantly in the lower (dorsal) half of the pelvic nerve networks and the motor nerves being distributed predominantly in the upper (ventral) half of the pelvic nerve networks.

The comparative urodynamic study carried out by F.Maneschi et al (27) on 15 patients showed a reduced detrusor activity observed during the filling phase, as expressed by the significant increase of the maximum cystomanometric capacity, and during the voiding phase, as expressed by the significant increase of flow time. The time to achieve spontaneous voiding with postvoid residual urine less than $50 \mathrm{~mL}$ was markedly reduced in nerve-sparing patients (4 days) than in patients treated with conventional radical hysterectomy (8 days), confirming that nerve sparing improves bladder function recovery immediately after surgery.

As postvoid residual urine volume is generally considered to be a valid index of bladder function after radical hysterectomy, patients are being encouraged to void with abdominal straining to increase voiding volume and decrease postvoid residual urine volume. Such guidance may cause long-term deterioration of bladder function, which has been reported to persist for 
10 years or more after $\mathrm{RH}$. Immediately after $\mathrm{RH}$ has been performed, not only voiding function but also storage function deteriorates. Storage function is represented by bladder compliance, a decrease in which results in continuous high pressure inside the bladder, which may in turn cause ischemia and injury to the detrusor muscles. Progressive hydronephrosis may follow deterioration of detrusor muscle function, selfcatheterization being needed (28).

Whever radiotherapy (RT) to the whole pelvis after $\mathrm{RH}$ causes further deterioration of bladder function is still controversial. Ralph et al (29) reported an adverse effect of postoperative RT on lower urinary function. Whereas bladder neck and urethral functions were similar in all groups, bladder compliance was significantly reduced after $\mathrm{RH}$ plus RT compared to that after $\mathrm{RH}$ alone (28).

The study of Oda et al (28) including type $4 \mathrm{RH}$ and urodynamic studies showed that a non-nerve-sparing procedure, adjuvant RT and voiding with abdominal pressure in the acute/subacute postoperative period were risk factors for prolonged deterioration of bladder compliance. This study revealed another important point: bladder function in the acute phase of the post$\mathrm{RH}$ period was greatly decreased regardless of whether a nerve-sparing procedure was performed or not. Thus, the prognosis of bladder function in the recovery phase can take two courses: recovery to preoperative quality or a permanent decrease in quality. To enhance recovery of bladder function, the patients are instructed to void without abdominal pressure. Thus, voiding with abdominal strain and adjuvant RT will prevent improvement in the recovery of bladder compliance in patients who have undergone $\mathrm{RH}$.

The meta-analyses of Kim et al (24)is meaningful in supporting the efficacy of autonomic nerve preservation by NSRS on urinary functions.

\section{Anorectal dysfunction after radical hysterectomy}

Anorectal dysfunctions have been reported in $5 \%$ to $10 \%$ of patients after CRS. Sympathetic nerves inhibit the expulsion of feces and stimulate the internal sphincter of the anus, whereas parasympathetic nerves show opposite effects (16,30). In 1991, Barnes W. et al (30) suggested the hypothesis that injury to the pelvic autonomic nerves by CRS disrupts the spinal reflex, which causes internal sphincter dysregulation and decreased rectal sensation.

Autonomic nerve damage during radical hysterectomy is thought to play a crucial role in long-term anorectal dysfunctions such as diarrhea, constipation, and fecal incontinence and several studies reported recently that NSRH confers a better clinical outcome in terms of anorectal dysfunctions. Cibula et al (25) assessed anorectal function six months after surgery with questionnaires by comparing patients who had undergone CRH with those who had undergone NSRH. The authors found that the morbidity of fecal incontinence was significantly lower in the NSRH group. In the study of Wang W. et al (18), with the median follow-up time of 31.9 months, both constipation and diarrhea were improved by NPSRH. An important study, the metaanalysis of Kim et al. (24) supports the hypothesis and suggests that NSRS may reduce the incidence of functional anorectal disorders, such as constipation.

However, long-term anorectaldysfunctions are frequently influenced by various factors including perioperative adjuvant radiotherapy or chemotherapy. Thus, the effect of NPSRH on anorectal function needs to be confirmed with further studies.

\section{Sexual dysfunctions}

Considerable sexual dysfunction, including decrease in sexual interest and orgasm, and vaginal dryness are also noticed after CRS, which compromise sexual activity and cause substantial distress (32).

The hypogastric nerves innervate the sexual response of the vagina: after sexual arousal, the blood flow in the vaginal wall increases, resulting in lubrication of the vagina.Reduced lubrication, leading to dyspareunia, is the most prominent complaint of women after radical surgery for cervical cancer. The increase in vaginal blood flow during sexual arousal can be measured using vaginal photoplethysmography. In the study of Pieterse et al (13), the increase in vaginal blood flow in reaction to erotic stimuli differed semnificantly betweenwomen after CRH compared with NSRH. The vaginal response, or increase in vaginal blood flow, of women after NSRH did not differ from the vaginal response of healthy controls, whereas the response of women after $\mathrm{CRH}$ was far less.

Patients undergoing open abdominal type $\mathrm{C} 2$ radical hysterectomyexperience worse sexual function than patients undergoing type $\mathrm{B}$ radical hysterectomy. Ditto et al. (22), in a large series of CC patients, suggested that open abdominal type $\mathrm{C} 1$ radical hysterectomy does not worsen sexual function in comparison with type B radical hysterectomy. In fact, the preservation of pelvic autonomic nerves during dissection of the parametrium is one of the most relevant factors reducing the occurrence of sexual dysfunction in patients undergoing type $\mathrm{C} 1$ radical hysterectomy.

Both conventional laparoscopic RH and NS-laparo- 
scopic RH impact negatively on patients' sexual function. However, the NS approach impairs sexual function less, minimizing the effects of radical surgery $(22,33)$.

In the meta-analysis conducted by H.S. Kim et al. (24) in 2015, including 2253 patientsfrom two randomized controlled trials, seven prospective and eleven retrospective cohort studies, a comparison of urinary, anorectal and sexual dysfunction between CRS and NSRS in terms of long-term outcomes ( 6 or 12 months after surgery) found that in NSRH operative time was longer and intraoperative complications were less common; the length of the resected vagina was shorter $(26,31)$. No significant differences were found in blood loss, hospital stay or postoperative complications. The lenght of the resected parametrium, DFS and OS were not significantly different. On the other hand, the safety of NSRS still remains controversial because of the concerns of less radicality of NSRS (34). Urinary incontinence and frequency, as well as anorectal dysfunctions were less common in NSRS. No signifiant differences were observed in sexual dysfunction between CRS and NSRS, which means that multiple factors, including vaginal shortness, tissue fibrosis, radiotherapy, a decrease in ovarian function and psychological factors may be more important to influence the sexual functions (18). The conclusion of the meta-analysis was that NSRS can give better quality of life preserving urinary and anorectal functions without adverse effects on clinical outcomes and sexual functions in patients with early-stage CC (24).

Similar results were obtained by Z.Liu et al. (35) evaluating the clinical efficacy and safety of laparoscopic NSRS for 120 patients with locally advanced CC and by Y.Longet al (3), in a systematic review and metaanalysis evaluating clinical efficacy and safety of nervesparing radical hysterectomy for cervical cancer.

As for the perspectives in nerve-sparing techniques and the attempt to optimize them, data about functional anatomy, computed assisted dissection and 3D reconstruction with immunohistochemical techniques are interestingly analised in the paper of Moszkovicz et al (36).

Recent papers also evaluate the solutions we might have after nerve injury, i.e. laparoscopic implantation of neural electrode (implant below the level of the injury) to restore the motor or autonomic function $(37,38)$.

\section{CONCLUSIONS}

Nerve-sparing radical hysterectomy is a reliable technique in the surgical treatment of cervical cancer. Available evidence suggests that it is better than conventional radical hysterectomy for postoperative recovery of pelvic organ function and postoperative morbidity, while the two techniques involve similar extent of resection and clinical safety. The quality of life in the nerve-sparing radical hysterectomy one year after the surgery is better compared with the conventional techniques, especially in social and family life, emotional well-being, working status. However, the results are based on a relatively small number of controlled trials, most of which are non-randomized, therefore these findings should be confirmed in larger, randomized clinical trials.

\section{REFERENCES}

1. Siegel RL, Fedewa SA, Miller KD, Goding-Sauer A, Pinheiro PS Martinez-Tyson D et al. Cancer statistics for Hispanics/Latinos, 2015. CA Cancer J Clin. 2015 Nov;65(6):457-480.

2. Puntambekar SP, Patil A, Joshi SN, Rayate NV, Puntambekar SS, Agarwal GA. Preservation of autonomic nerves in laparoscopic total radical hysterectomy. J LaparoendoscAdvSurg Tech A. 2010 Dec; 20(10):813-819.

3. Long Y, Yao DS, Pan XW, Ou TY. Clinical efficacy and safety of nervesparing radical hysterectomy for cervical cancer: a systematic review and meta-analysis. PLoS One. 2014:9(4):e94116-

4. Okabayashi H. Radical hysterectomy for cancer of the cervix uteri. Modification of the Takayama operation. SurgGynecol Obstet. 1921;33:335-341.

5. Piver MS, Rutledge F, Smith JP. Five classes of extended hysterectomy for women with cervical cancer. Obstet Gynecol. 1974 Aug; 44(2): 265-272.

6. QuerleuD,Morrow CP. Classification of radical hysterectomy. Lancet Oncol. 2008 Mar;9(3):297-303.

7. Yamaguchi K, Kobayashi M, Kato T, Akita K. Origins and distribution of nerves to the female urinary bladder: new anatomical findings in the sex differences. Clin Anat. 2011 Oct;24(7):880-885.

8. Mantzaris G, Rodolakis A, Vlachos G, Athanasiou S, Theocharis S, Sotiripoulou $\mathrm{C}$ et al. Magnifying lenses assisted nerve-sparing radical hysterectomy and prevention of nerve plexus trauma. Int $\mathrm{J}$ Gynecol Cancer. 2008 Jul;18(4):868-875.

9. Ercoli A, Delmas V, Gadonneix P, Fanfani F, Villet R, Paparella P et al. Classical and nerve-sparing radical hysterectomy: an evaluation of the risk of injury to the autonomous pelvic nerves. SurgRadiol Anat. 2003 Jul;25(3-4):200-206.

10. Mauroy B, Demondion X, Bizet B, Claret A, Mestdagh P, Hurt C. The female inferior hypogastric (= pelvic) plexus: anatomical and radiological description of the plexus and its afferences--applications to pelvic surgery. SurgRadiol Anat. 2007 Feb;29(1):55-66.

11. Hockel M. Basic neuroanatomy and neurophysiology of the urethrovesical function with special reference to extended hysterectomy. CME J GynaecolOncol 2002;7:32-5.

12. Sakuragi N, Todo Y, Kudo M, Yamamoto R, Sato T. A systematic nervesparing radical hysterectomy technique in invasive cervical cancer for preserving postsurgical bladder function. Int J Gynecol Cancer. 2005 Mar;15(2):389-397.

13. Pieterse QD, TerKuile MM, Deruiter MC, Trimbos JB, Kenter GG, Maas CP. Vaginal blood flow after radical hysterectomy with and without nerve sparing. A preliminary report. Int J Gynecol Cancer. 2008 May;18(3):576-583

14. Kavallaris A, Hornemann A, Chalvatzas N, Luedders D, Diedrich K, Bohlmann MK. Laparoscopic nerve-sparing radical hysterectomy: description of the technique and patients' outcome. Gynecol Oncol. 2010 Nov;119(2):198-201

15. Liang Z, Chen Y, Xu H, Li Y, Wang D. Laparoscopic nerve-sparing radical hysterectomy with fascia space dissection technique for cervical cancer: description of technique and outcomes. Gynecol Oncol. 2010 Nov;119(2):202-207. 
16. Trimbos JB, Maas CP, Deruiter MC, Peters AA, Kenter GG. A nervesparing radical hysterectomy: guidelines and feasibility in Western patients. Int J Gynecol Cancer. 2001 May;11(3):180-186.

17. Li B, Li W, Sun YC, Zhang R, Zhang GY, Yu GZ et al. Nerve planesparing radical hysterectomy: a simplified technique of nerve-sparing radical hysterectomy for invasive cervical cancer. Chin Med J (Engl ). 2011 Jun;124(12):1807-1812.

18. Wang W, Li B, Zuo J, Zhang G, Yang Y, Zeng $\mathrm{H}$ et al. Evaluation of pelvic visceral functions after modified nerve-sparing radical hysterectomy. Chin Med J (Engl ). 2014;127(4):696-701.

19. Kanao H, Fujiwara K, Ebisawa K, Hada T, Ota Y, Andou M. Various types of total laparoscopic nerve-sparing radical hysterectomies and their effects on bladder function. J GynecolOncol. 2014 Jul;25(3):198205.

20. Hockel M, Horn LC, Manthey N, Braumann UD, Wolf U, Teichmann G et al. Resection of the embryologically defined uterovaginal (Mullerian) compartment and pelvic control in patients with cervical cancer: a prospective analysis. Lancet Oncol. 2009 Jul;10(7):683-692.

21. Benedetti-Panici P, Zullo MA, Plotti F, Manci N, Muzii L, Angioli R. Long-term bladder function in patients with locally advanced cervical carcinoma treated with neoadjuvant chemotherapy and type 3-4 radical hysterectomy. Cancer. 2004 May 15;100(10):2110-2117.

22. Ditto A, Martinelli F, Hanozet F, Reato C, Solima E, Zanaboni F et al. Class III NSRH: oncological outcome in 170 cervical cancer patients. GynecolOncol. 2010 Nov;119(2):192-197.

23. van den Tillaart SA, Kenter GG, Peters AA, Dekker FW, Gaarenstroom KN, Fleuren GJ et al. Nerve-sparing radical hysterectomy: local recurrence rate, feasibility, and safety in cervical cancer patients stage IA to IIA. Int J Gynecol Cancer. 2009 Jan;19(1):39-45.

24. Kim HS, Kim K, Ryoo SB, Seo JH, Kim SY, Park JW et al. Conventional versus nerve-sparing radical surgery for cervical cancer: a metaanalysis. J GynecolOncol. 2015 Apr;26(2):100-110.

25. Cibula D, Slama J, Velechovska P, Fischerova D, Zikan M, Pinkavova I et al. Factors affecting spontaneous voiding recovery after radical hysterectomy. Int J Gynecol Cancer. 2010 May;20(4):685-690.

26. Possover M, Stober S, Plaul K, Schneider A. Identification and preservation of the motoric innervation of the bladder in radical hysterectomy type III. GynecolOncol. 2000 Nov;79(2):154-157.

27. Maneschi F, laniri P, Sarno M, Gagliardi F, Panici PB. Nerve-sparing class III-IV radical hysterectomy: urodynamic study and surgical technique. Int J Gynecol Cancer. 2012 May;22(4):675-680.

28. Oda Y, Todo Y, Hanley S, Hosaka M, Takeda M, Watari H et al. Risk factors for persistent low bladder compliance after radical hysterectomy. Int J Gynecol Cancer. 2011 Jan;21(1):167-172.

29. Ralph G, Tamussino K, Lichtenegger W. Urological complications after radical hysterectomy with or without radiotherapy for cervical cancer. Arch Gynecol Obstet. 1990;248(2):61-65.

30. Barnes W, Waggoner S, Delgado G, Maher K, Potkul R, Barter J et al. Manometric characterization of rectal dysfunction following radical hysterectomy. GynecolOncol. 1991 Aug;42(2):116-119.

31. Raspagliesi F, Ditto A, Fontanelli R, Zanaboni F, Solima E, Spatti $G$ et al. Type II versus Type III nerve-sparing radical hysterectomy: comparison of lower urinary tract dysfunctions. GynecolOncol. 2006 Aug; 102(2):256-262.

32. Bergmark K, Avall-Lundqvist E, Dickman PW, Henningsohn L, Steineck $G$. Vaginal changes and sexuality in women with a history of cervical cancer. N Engl J Med. 1999 May 6;340(18):1383-1389.

33. Bogani G, Serati M, Nappi R, Cromi A, di Naro E, Ghezzi F. Nervesparing approach reduces sexual dysfunction in patients undergoing laparoscopic radical hysterectomy. J Sex Med. 2014 Dec;11(12): 3012-3020.

34. Dursun P, LeBlanc E, Nogueira MC. Radical vaginal trachelectomy (Dargent's operation): a critical review of the literature. Eur J Surg Oncol. 2007 Oct;33(8):933-941.

35. Liu Z, Li X, Tao Y, Li W, Yang Y, Yao Y et al. Clinical efficacy and safety of laparoscopic nerve-sparing radical hysterectomy for locally advanced cervical cancer. Int J Surg. 2016 Jan;25:54-58.

36. Moszkowicz D, Alsaid B, Bessede T, Penna C, Benoit G, Peschaud F. Female pelvic autonomic neuroanatomy based on conventional macroscopic and computer-assisted anatomic dissections. Surg Radiol Anat. $2011 \mathrm{Jul} ; 33(5): 397-404$.

37. Possover M, Schurch B, Henle KP. New strategies of pelvic nerves stimulation for recovery of pelvic visceral functions and locomotion in paraplegics. NeurourolUrodyn. 2010 Nov;29(8):1433-1438.

38. Rabischong B, Larrain D, Rabischong P, Botchorishvili R, Fraisse G, Gallego $S$ et al. Laparoscopic implantation of neural electrodes on pelvic nerves: an experimental study on the obturator nerve in a chronic minipig model. SurgEndosc. 2011 Nov;25(11):3706-3712. 